\title{
LA INCLUSIÓN DE CLÁUSULAS SOCIALES EN LA CONTRATACIÓN PÚBLICA EN EL MARCO DEL POSTCONFLICTO COLOMBIANO
}

\author{
THE INCLUSION OF SOCIAL CLAUSES IN PUBLIC HIRING IN THE FRAMEWORK \\ OF THE COLOMBIAN POSTCONFLICT
}

Juan David Duque Botero

\begin{abstract}
Abogado y magíster en Derecho Administrativo de la Universidad del Rosario, experto en Contratación Pública de la Universidad de Deusto y doctor en Derecho de la Universidad Alfonso X El Sabio de Madrid (España); se ha desempeñado como secretario general de la Superintendencia de Industria y Comercio, magistrado auxiliar de la Sección Tercera del Consejo de Estado, subsecretario de Planeación Distrital. Es profesor titular de la cátedra de Contratación Estatal, Facultad de Jurisprudencia y en la especialización de Derecho Administrativo, de la Universidad del Rosario. Actualmente es el secretario general del Ministerio de Tecnologías de la Información y las Comunicaciones. E-mail: jduque@duquebotero.com
\end{abstract}

Convidado

Doi: $10.5585 / \mathrm{rdb} . v 17 \mathrm{i} 7.709$

RESUMEN: La contratación estatal tuvo su definición alterada en diversos momentos a lo largo del tiempo, de acuerdo con los intereses y/o las deficiencias estatales. En los días actuales, la definición encuentra guarida en la doctrina de las cláusulas sociales, como complemento de las cláusulas principales de los contratos, sin suprimirlas. Las referidas cláusulas sociales englobarían intereses ambientales, sociales y éticos. Se entiende que a partir de ese nuevo contexto, la contratación pública representaría una importante herramienta para traer más eficiencia a la actuación estatal, así como mejorar la competitividad en el mercado público, mientras que paralelamente se relacionaría con un proyecto de construcción de paz actualmente en vigor en Colombia, permitiendo así un continuo proceso de reinserción social, principalmente de las Fuerzas Armadas Revolucionarias de Colombia (FARC) y del Ejército de Liberación Nacional (ELN). En este sentido, el presente artículo tiene el propósito analizar el tercer punto desarrollado en la agenda de la Habana y quinto punto desplegado en Ecuador.

Palabras-claves: Cláusulas Sociales; Contratación Pública; Instrumento de Política Económica; Postconflicto Colombiano.

ABSTRACT: State bidding had its definition changed at various times over time, in accordance with State interests and/or deficiencies. In the present day, the definition finds shelter in the doctrine of social clauses, as a complement to the main clauses of the contracts, without suppressing them. These social clauses would encompass environmental, social and ethical interests. It is understood that from this new context, public procurement would represent an important tool to bring more efficiency to State activities, as well as to improve the competitiveness in the public market, while in parallel it would be related to a project of construction of peace currently in place in Colombia, thus allowing a continuous process of social reintegration, mainly of FARC and ELN. In this sense, the present article has the purpose to analyze the third point developed in the agenda of Havana and fifth point deployed in Ecuador.

Keywords: Social Clauses; Public Hiring; Instrument of Economic Policy; Colombian Post conflict. 
SUMÁRIO: 1. La contratación pública como instrumento de política económica. 2. Los acuerdos, paz, reinserción y reintegración. 3. La Contratación Pública como Instrumento de Paz y Reconciliación en el Marco del Postconflicto. Bibliografía.

\section{LA CONTRATACión PÚBLICA COMO INSTRUMENTO DE POLÍTICA ECONÓMICA}

La definición tradicional de la contratación estatal ha mutado en diversos momentos como una respuesta a las variantes y siempre dispares insuficiencias del Estado. Actualmente, puede entenderse por ella aquel instrumento público a partir del cual se dota a las entidades gubernamentales de obras, bienes y servicios que satisfagan las necesidades propias del funcionamiento del aparato estatal, el cumplimiento de los fines e intereses públicos y simultáneamente coadyuva a la satisfacción de necesidades agrupadas a partir de la doctrina de las cláusulas sociales ${ }^{1}$ en tres grandes grupos: ambientales, sociales y éticos.

La inclusión de cláusulas sociales en los procesos contractuales emprendidos por la Administración representa un mecanismo apropiado para lograr eficiencia en el gasto público y una herramienta con potencialidad para cumplir con estándares responsables impuestos a la actividad estatal. Así, a pesar que en el país la inclusión de este tipo de preceptos no tiene gran desarrollo, su implementación es capaz de promover conductas benéficas en la sociedad, mejorar los niveles de competitividad en el mercado público y garantizar un mejor uso de los recursos públicos.

No sobra advertir que la normatividad colombiana no hace ninguna referencia que permita intuir la restricción, prohibición o limitación en la introducción de este tipo de prerrogativas en derecho contractual público, aunque sí debe mencionarse que su despliegue no podrá, bajo ningún respecto vulnerar el núcleo esencial del principio de igualdad u obviar el objeto contractual que pretende satisfacerse con el proceso de compra ${ }^{2}$.

El ideal busca incluir criterios que complementen las cláusulas principales del contrato y que por tanto dependan de ellas, redactadas de forma que no entorpezcan la labor primaria del contrato y simultáneamente generen nuevas cargas obligacionales que repercutan en la elección de la oferta más favorable y en los raciocinios que influyen para la adjudicación del contrato.

Ya que el esquema normativo del país permite un uso relativamente amplio de la discrecionalidad que se despliega sobre la actividad estatal, a partir del cual pueden desarrollarse políticas públicas internas que permitan satisfacer objetivos constitucional, legal y normativamente establecidos, la introducción de cláusulas sociales con la intención de fomentar los proyectos de construcción de paz en el país, resultan ser un objetivo plenamente legítimo. Con la incorporación de nuevas cláusulas a los documentos expedidos en ejercicio de la función contractual del Estado, la Administración es capaz de aportar una nueva herramienta que se despliegue en el proceso de reintegración de los excombatientes.

No es desconocido que actualmente Colombia está atravesando por uno de los procesos más trascendentales que se han desplegado en su historia. Los avances que han evidenciado en

\footnotetext{
${ }^{1}$ Las cláusulas sociales deben ser entendidas como aquellas directrices que "pueden incluirse como requisito previo (criterio de admisión), como elemento de valoración (criterio de adjudicación) o como una obligación de ejecución inherente al contrato (condición en fase de ejecución). Del mismo modo, el contenido de las cláusulas puede ser muy variado, pero siempre tendrá como último objetivo la persecución y consecución de un fin social" (Torres Mena, 2015, p. 24).

${ }^{2} \mathrm{Al}$ respecto, Gimeno ha ratificado esta posición determinado que las tres formas en que se pueden desarrollar las cláusulas sociales, pueden servir para determinar la oferta económicamente más ventajosa, en aquellos casos en que se suponga una ventaja económica para la entidad contratante que pueda atribuirse al producto o servicio objeto de la licitación. Pero, deberán estar relacionados -aun de forma indirecta- con el objeto del contrato y no con la cualificación del contratista, pues ese aspecto se debe valorar como un procedimiento restringido (Gimeno, 2013, p. $68)$.
} 
los diálogos y la firma de acuerdos entre El Gobierno Nacional, las Fuerzas Armadas Revolucionarias de Colombia (FARC) y el Ejército de Liberación Nacional (ELN), han configurado lo que podría ser una nueva era para un país que ha enfrentado los efectos de una guerra que supera los 50 años y actualmente puede posicionar a las guerrillas que se crearon y fortalecieron en este periodo, como las más antiguas del mundo.

La perspectiva de un país que desarrolle sus modelos sociales en un escenario de pacífico y de reconciliación, permite contemplar una gama de posibilidades limitadas en otras circunstancias. El proceso hacia un Estado en paz requiere la intervención de todos los sectores de la sociedad, y por supuesto la intervención activa de la empresa privada, como intermediaria idónea en la etapa de estabilización de personas que se hayan desmovilizado de los grupos armados que se han registrado en el país, víctimas y militares.

Según los pronunciamientos del Alto Comisionado de las Naciones Unidas para los Derechos Humanos, en un proceso de desarme, desmovilización y reintegración como el emprendido por Colombia, no es suficiente con la firma de los acuerdos de paz ya que esta etapa tiene un alcance restringido y sus consecuencias directas no permiten garantizar la no repetición. Por ello, una vez agotados los alcances máximos de las primeras fases del proceso de paz, es indispensable el despliegue de un esquema capaz de sustentar, atender y enfrentar las necesidades que surgen en el marco del postconflicto.

Frente a dicho panorama, Colombia intenta implementar una serie de Políticas Públicas, desde las cuales espera superar y mitigar sino todas, parte de las consecuencias que ha dejado y continua reproduciendo el conflicto armado, a modo de ilustración puede hacerse referencia a la ley de víctimas, los procesos de restitución de tierras, los proyectos de justicia transicional, la construcción de un marco jurídico para la Paz y el Programa de Desarme, Desmovilización y Reintegración (DDR), en donde se encuentra inmersa la Política de Reintegración Social y Económica de Personas y Grupos Alzados en Armas (PRSE), propuestas políticas que más allá de su pertinencia son nombradas en esta investigación como referentes de una respuesta política nacional frente a unos fenómenos que hoy experimenta la sociedad colombiana (Mejía Gómez, 2014, p. 8).

En ese orden de ideas, el presente escrito tomará el tercer punto desarrollado en la agenda de la Habana ${ }^{3}$ y quinto punto desplegado en Ecuador, como fundamento de las teorías que desarrollará más adelante con lo que tiene que ver con la contratación estatal y su eventual relación con el proyecto de construcción que paz que iniciará próximamente en el país. El estudio no tiene la capacidad de permear los cinco temas que se han propuesto en la mencionada agenda, el documento centrará su atención en aquel acápite que permite dos grandes contribuciones por parte de la contratación estatal, ellas son: el establecimiento de un marco garantista que reduzca las posibilidades de repetición y la disposición de un esquema que permita un proceso de reinserción social sin mayores traumatismos para los individuos involucrados.

Dentro del discurso político que ha pronunciado el Presidente de la República durante los últimos meses se ha evidenciado que dentro de las metas claras que se han establecido a este respecto resaltan aquellas relacionadas con el cese al fuego y las hostilidades bilaterales de forma

\footnotetext{
${ }^{3}$ Dentro de la agenda propuesta en desarrollo de los diálogos emprendidos entre el Gobierno Nacional y las FARCEP, se propusieron 5 puntos básicos, ellos fueron: i) Reforma Económicas (desarrollo agrario), ii) Participación Política de la Guerrilla, iii) Fin del conflicto armado, iv) Fin del Narcotráfico, v) Derechos de las víctimas. Por su compatibilidad, el texto se centrará en el tercer punto para desarrollar la teoría que presentará. En lo que hace a los diálogos desarrollados entre los negociadores del Gobierno Nacional y el ELN, hasta el momento se han propuesto 6 puntos de necesaria discusión: i) Participación en la sociedad, ii) Democracia para la paz, iii) Transformaciones para la paz, iv) Víctimas, v) Fin del conflicto vi) implementación de lo acordado.

Revista de Direito Brasileira | São Paulo, SP | v. 17 | n. 7 | p. 63 - 75 | Mai./Ago. 2017
} 
definitiva, la dejación de armas y la reincorporación de las guerrillas en la vida civil, con las garantías necesarias para avalar condiciones sociales, políticas y económicas mínimas a corto, mediano y largo plazo.

Este último paso, caracterizado por la Comunidad Internacional, como aquella etapa en la cual se despliegan los procesos de reinserción y reconciliación de la sociedad civil, involucra un esfuerzo desplegado desde todos los sectores que conforman la sociedad colombiana y es por ello que requiere un marco mucho más exigente que el desarrollado de forma interdisciplinaria e interdependiente en otras etapas ${ }^{4}$. "Este esfuerzo debe ser asumido como uno común, en donde el Gobierno, el Estado, el sector privado y la sociedad civil tengan la posibilidad de contribuir enormemente" (Reyes Rodríguez, 2015, p. 232).

Debe hacerse énfasis en el rol que desempeña el Estado al utilizar las compras públicas como herramienta de política económica y social, en atención al volumen de compras que realiza. De ahí, la viabilidad de un proyecto que busque la ejecución de metas que fomenten un esquema de inclusión laboral introducido, en principio, en la etapa precontractual y durante la definición de obligaciones de ejecución del contrato, para revestirlas como instrumentos idóneos en la consecución de algunos de los objetivos planteados para el desarrollo del proceso postconflicto que atravesará Colombia.

Ahora bien, ya que según el artículo $3^{\circ}$ de la Ley 80 de 1993 "los funcionarios públicos tendrán en consideración que al celebrar contratos y con la ejecución de los mismos, las entidades buscan el cumplimiento de los fines estatales, la continua y eficiente prestación de los servicios públicos y la efectividad de los derechos e intereses de los administrados que colaboran con ellos en la consecución de dichos fines" y que según el artículo el artículo $2^{\circ}$ de la Constitución Política colombiana "son fines esenciales del Estado: servir a la comunidad, promover la prosperidad en general y garantizar la efectividad de los principios, derechos y deberes consagrados en la Constitución, facilitar la participación de todos en las decisiones que los afectan y en la vida económica, política, administrativa y cultural de la Nación, mantener la integridad territorial y asegurar la convivencia pacífica y la vigencia de un orden justo" no resulta alejado al contexto social actual incentivar en los servidores públicos el ideal de redactar en sus pliegos de condiciones parámetros que generen un valor agregado para el proceso de reinserción de los grupos armados a la vida civil, lo cual entre otras cuestiones permitiría, a mediano plazo, prescindir de auxilios económicos y tratamientos especiales.

En cuanto al preámbulo de la carta debe mencionarse, también, que se expone de forma clara como "el pueblo de Colombia, en ejercicio de su poder soberano, representado por sus delegatarios a la Asamblea Nacional Constituyente invocando la protección de Dios, y con el fin de fortalecer la unidad de la Nación y asegurar a sus integrantes la vida, la convivencia, el trabajo, la justicia, la igualdad, el conocimiento, la libertad y la paz, dentro de un marco jurídico, democrático y participativo que garantice un orden político, económico y social justo, y comprometido a impulsar la integración de la comunidad latinoamericana". A partir de allí, se adoptan una serie de compromisos necesarios para la satisfacción del espíritu del Estado proclamado por el constituyente primario, y consecuencialmente, se vincula la actividad pública con intención de garantizar y satisfacer los fines estatales preestablecidos por la Carta Política y las demás normas pertinentes, en el marco de los procesos de paz desplegados el país. Descripción que ineludiblemente representa el deber de utilización de todos aquellos medios con los que cuente el Estado para fomentar e impulsar las políticas del postconflicto.

Se pretende, entonces, incluir cláusulas sociales con objetivos enfocados a soportar las necesidades del postconflicto en el contrato estatal, para que, mediante deliberaciones y

\footnotetext{
${ }^{4}$ Aunque su carácter secuencial e interdependiente exige abordar cada fase con la misma rigurosidad, la reinserción y en especial la reintegración, como un todo, demandan particulares esfuerzos institucionales que trascienden la acción del gobierno e involucran a toda la sociedad como catalizadora de una efectiva reincorporación y reconciliación de los antiguos combatientes (Fundación ideas para la paz, 2014, p. 51).
}

Revista de Direito Brasileira | São Paulo, SP | v. 17 | n. 7 | p. 63 - 75 | Mai./Ago. 2017 
raciocinios lógicos, el funcionario público justifique estimaciones diversas a las inherentes al contrato sin que ello represente una barrera de entrada para eventuales oferentes. La cuestión se circunscribe a una forma de compra avalada por contenidos socialmente aceptados ${ }^{5}$, fundamentada en un estricto deber de observancia de los principios de prevalencia del interés general sobre el particular y libre competencia entre sectores económicos, y, por supuesto, la preservación y satisfacción de derechos de segunda generación, la ineludible necesidad de garantizar el principio de igualdad en todas las esferas que se despliegan en el Estado colombiano y el establecimiento de esquemas capaces de fortalecer la prosperidad y los sistemas económicos desarrollados en los mercados nacionales.

Estas transformaciones tienen que ver con la consolidación del Estado Social de Derecho en todo el territorio nacional, como condición para la garantía y la protección de los derechos civiles y libertades fundamentales de todos los ciudadanos, así como la gradual y progresiva garantía de sus derechos sociales; la integración regional del país, como condición para la promoción de la igualdad económica y social y la superación de las brechas y desequilibrios territoriales; el aumento de la calidad en la educación; así como mecanismos institucionales para la gestión y transformación de conflictos sin violencia priorizando el diálogo frente a la estigmatización y la violencia, así como el predominio y generalización en Colombia de valores y cultura de paz y democracia (Gobierno de Colombia, 2014 p. 12).

Sentado esto, debe mencionarse que ya que los propósitos perseguidos por la ponencia han circunscrito el asunto a desarrollar sobre los efectos que puede tener un esquema implementado en la contratación estatal sobre los procedimientos de reintegración social que se llevarán a cabo con los miembros de los grupos armados asentados en Colombia, debe exponerse un breve estudio que permita entender esta etapa y sus repercusiones reales en la sociedad colombina.

\section{DE LOS ACUERDOS, PAZ, REINSERCIÓN Y REINTEGRACIÓN.}

Según las conclusiones que expone la Universidad de Uppsala (Suecia) a partir de su estudio sobre los acuerdos de paz desplegados entre 1975 y 2011, los postulados de Desarme, Desmovilización y Reintegración (DDR), resultan ser una pieza transversal y esencial en los procesos de paz que pretendan desplegarse en cualquier contexto.

Ya que existe un universo de particularidades en cada proceso emprendido y que cada iniciativa se encuentra adscrita a circunstancias socio-temporales específicas, no deben proponerse fórmulas genéricas para cada caso, aunque, sí existen ciertos estándares mínimos tendientes a soportar un proceso justo, equitativo e idóneo; propuestos por el grupo de trabajo inter-agencial sobre Desarme, Desmovilización y Reintegración (Cfr. Estándares Integrados de DDR de Naciones Unidas (Siglas en inglés IDDRS/2004). Y, adicionalmente, existen diversas directrices que compilan una serie de prerrogativas que aportan los elementos necesarios para la creación, estructuración y desarrollo de los postulados de DDR y han sido el resultado de lecciones aprendidas y propuestas fallidas y exitosas en otros ámbitos.

En ese entendido, como primera medida debe exponerse que las políticas de DDR deben ser destinadas a cinco grandes grupos de personas: i) Hombres y mujeres combatientes, ii) Niños, adolescentes y jóvenes desvinculados de grupos armados, iii) Personas no combatientes asociadas a grupos armados, iv) Discapacitados y heridos, y v) Familiares de combatientes. (Cfr. Fisas,

\footnotetext{
${ }^{5}$ La compra social de que trata este texto considera aspectos como la accesibilidad y calidad del empleo, la perspectiva de género, la contratación de personas con discapacidad o la contratación de empresas de inserción y centros especiales de empleo. (Medina Jurado, 2010).
}

Revista de Direito Brasileira | São Paulo, SP | v. 17 | n. 7 | p. 63 - 75 | Mai./Ago. 2017 
2011). Sin embargo, por las condiciones que plantea el documento, las políticas que pretendan incluir cláusulas sociales en favor del postconflicto colombiano, deberán centrarse en hombres y mujeres combatientes, personas no combatientes asociadas a grupos armados, familiares de combatientes y víctimas, en edad para trabajar.

Lo anterior ya que, en Colombia, al igual que en otros escenarios similares, es importante que durante la fase de reinserción no sólo se tenga en cuenta a los combatientes y civiles de apoyo, sino que también se evidencien esfuerzos para acompañar y garantizar un proceso de inclusión y soporte a familiares perjudicados y a las comunidades receptoras (Cfr. Collin (et al), 2004).

Como segunda medida, cabe resaltar que un proceso idóneo de Desarme, Desmovilización y Reintegración tiene un promedio de 3 a 5 años, aunque esta cifra está sujeta a las condiciones contextuales de cada proceso. En este sentido las primeras fases (el Desarme y la Desmovilización) toman aproximadamente un año, mientras que la Reinserción absorbe el resto del tiempo proyectado. (Cfr. Caramés, 2006). Sin perjuicio de lo anterior, el proyecto de reintegración propuesto para el caso colombiano, se extenderá por un periodo aproximado de seis años y medio en los cuales, se busca desarrollar las competencias y capacidades de los reinsertados en los entornos sociales en los cuales se desarrollarán en el futuro, una de la cuales se orienta a la inclusión social y laboral de este grupo.

Por los avances en las conversaciones desplegadas entre las guerrillas de las FARC y el ELN en los dos últimos años, las iniciativas y los proyectos que buscan favorecer un postconflicto idóneo se han convertido en el epicentro de la actividad estatal. Y su despliegue representará grandes y variados esfuerzos. Es por ello que, como última medida, el proceso debe observar dos elementos estructurales para garantizar un mecanismo eficiente que satisfaga los objetivos perseguidos a partir de las etapas de DDR, ellos son:

a. La disposición de mecanismos que garanticen un proceso de reinserción económica, social y política

b. La disposición de mecanismos que aseguren la no repetición ${ }^{6}$.

Ambas iniciativas permiten subsumirse en la presente propuesta y sus efectos pueden verificarse en tiempos más ciertos. Siguiendo el modelo propuesto por la Organización de Naciones Unidas -ONU-, el desarme se expondrá como la primera fase del proceso de DDR. Las características que identifican este momento se adaptan a las condiciones del conflicto colombiano, pues bajo las circunstancias en las cuales se efectuaron el proceso de paz con las FARC-EP en los diálogos de La Habana, finalizados en por la firma del acuerdo final el 24 de noviembre de 2016, y los esfuerzos evidenciados en Ecuador, con los negociadores del Ejército de Liberación Nacional -ELN-, no podrían otorgarse títulos de victoria y derrota a ninguna de las partes del conflicto.

Bajo las condiciones de un de un proceso cooperativo, las partes del proceso colombiano han desarrollado voluntariamente los postulados de cuatro fases propuestas por Johanna Spear en su libro Disarmament and Demobilization, y que deberán responder a los principios de legitimidad, transparencia, control, seguridad y sostenibilidad; estas son: i) Recolección de información y planeamiento operativo, ii) Recolección de armas, iii) Almacenamiento, iv) Destrucción.

Ya que el objetivo de este texto no se justifica en todos postulados del DDR, no se ahondará más en el Desarme, aunque no sobra advertir que además de las repercusiones políticas

\footnotetext{
${ }^{6}$ Los factores que deben limitarse que por el riesgo que su despliegue representa para la reincidencia son: i. dificultades económicas de toda índole; ii. Acciones discriminatorias en contra de aquellas personas que buscan reintegrarse; iii. Impedimentos para la participación en escenarios políticos; iv. intentos de sabotaje por parte de la oposición y v. ausencia de presencia estatal (Cfr. Nussio, 2009).
} 
y bélicas que representa, uno de los efectos más relevantes está dada por el simbolismo social que su realización conlleva, no solo por personificar el fin del conflicto, sino porque encarna la reasunción monopólica de la fuerza a cargo del Estado y uno de los actos simbólicos en favor de las víctimas que deja atrás el conflicto.

La desmovilización, al igual que la etapa anterior se desarrollará de manera superflua por no constituir el epicentro de estudio. Esta etapa, que suele desplegarse de forma casi simultánea con la primera tiene su fundamento en la desarticulación de la estructura bélica que se desarrolló al margen de la legalidad lo que incluye estructuras destinadas al combate y la conformación de redes de apoyo.

Dentro de los principales retos que enfrenta el gobierno nacional respecto de este ítem se encuentran las diversas garantías que deben ofrecerse a tanto a los desmovilizados como a aquellas personas que por los vínculos desarrollados (tanto afectivos, como bélicos) se encuentren en situación de vulnerabilidad. Así como la necesidad de crear condiciones idóneas que no permitan la reincidencia en prácticas violentas.

En cuanto a la reinserción y reintegración, deben hacerse algunas precisiones antes de abordar el tema de las cláusulas sociales que, por su naturaleza, se desarrollan a partir de esta arista del programa de DDR.

Los primeros proyectos que contemplaban estas tres circunstancias, tuvieron sus inicios restando importancia a esta última fase del programa, exponiendo la idoneidad del esquema con la satisfacción de las dos primeras etapas. Sin embargo, las amargas experiencias vividas en países como Samoa y Papúa demostraron que era necesario estructurar un plan en cuyo desarrollo se bloquearan las consecuencias negativas a largo plazo, permitiera reestructurar las bases de sociales de contextos fragmentados por la guerra y garantizará condiciones óptimas que imposibilitaran o al menos redujeran la configuración de elementos que permitieran la reincidencia violenta.

Ya que la reinserción comporta un primer acercamiento social que aquellas personas que a través de estos procesos buscan espacios en la comunidad, sus efectos deben determinarse a corto plazo y se limitan a kits de ayuda, auxilios económicos, reubicaciones temporales y demás elementos limitados en el tiempo que buscan iniciar un proceso de estabilización, condiciones que poco podrían desarrollarse desde el plano de la contratación estatal.

Después de la desmovilización, los combatientes se quedan sin sus medios habituales de ingresos o de supervivencia. Durante este período inicial, llamado de reinserción, hay que proporcionar a los excombatientes y a sus familias los medios necesarios para vivir dignamente, satisfaciendo sus necesidades básicas. Para los menores-soldado, lo importante es proporcionarles escolaridad en sus comunidades de origen y lo más rápidamente posible. Su estancia en los campos de acantonamiento ha de ser breve. No es conveniente que los menores reciben retribuciones monetarias. De la misma forma, las mujeres han de recibir un tratamiento diferenciado, tanto en esta etapa como en posterior, ya que han de crearse programas de reintegración específicos para ellas (Escola de Cultura de Pau, 2011).

La reintegración por otra parte, despliega un nuevo escenario de acción. Sus postulados desarrollan proyectos a mediano y largo plazo tendientes a restaurar las condiciones socioeconómicas de los excombatientes, sus familias y aquellas personas que desarrollaban labores como redes de apoyo para las guerrillas.

Esta última fase, llamada reintegración como una forma de suscribir sus alcances a las consecuencias socio-culturales del proceso, se convierte en una necesidad vital en los procesos de paz y representa uno de los ítems cuya logística y estructuración simbolizan mayores retos para el Estado. 
Se trata, entonces, de un proceso que busca dotar a los excombatientes y a los particulares que hicieron parte del conflicto armado, de un marco integral conformado por disposiciones legales, políticas, sociales y económicas que les permita regresar en condiciones dignas y lejos de cualquier tipo de hostilidad a la comunidad de la que se apartaron.

Contemplar la Reintegración Social y Económica de excombatientes de grupos armados al margen de la ley, implica considerar las diversas variables externas e internas que influyen en el desarrollo de una política de este tipo, variables relacionadas con las condiciones estructurales que tiene el Estado para enfrentar ese reto, en donde son relevantes aspectos como la igualdad, la justicia, la equidad, el desarrollo económico, la superación de la pobreza, el acceso a derechos fundamentales de los ciudadanos, la solidez institucional y la seguridad entre otros aspectos que sin duda inciden en el éxito o fracaso de una medida como la oferta de la reintegración social de personas alzadas en armas (Mejía Gómez, 2014, p. 9).

Debe tenerse presente que todas las iniciativas que se presenten en este ámbito, están restringidas por un marco temporal limitado. Razón por la cual todos los proyectos propuestos deben demostrar esfuerzos por consolidar circunstancias futuras que permitan a este grupo garantizar niveles estables de vida por sus propios medios.

\section{LA CONTRATACIÓN PÚBLICA COMO INSTRUMENTO DE PAZ Y RECONCILIACIÓN EN EL MARCO DEL POSTCONFLICTO}

La actividad privada adquiere nuevos e innumerables retos respecto al proyecto de paz que se encuentra atravesando el país. En efecto, los aportes que puedan evidenciarse por parte de este sector repercuten directamente en la conformación de la tan necesaria fuente sostenible, constante y estable de ingresos y formación ideada por el Estado.

Actualmente Colombia vive un proceso de desmovilización dentro del cual hay un programa de reintegración social, a través del cual los desmovilizados logran vincularse y reingresar a la sociedad civil a través de diferentes canales. Uno de estos canales es la inclusión laboral de estas personas. Sin embargo, este ámbito en concreto ha sido muy complejo pues existe una tendencia a imponer una barrera por parte de las empresas del sector privado de vincular personas desmovilizadas dentro de su nómina. Lo anterior ha generado que los desmovilizados retomen las armas y el resultado es más violencia. Por ello, es necesario que el Estado desarrolle una serie de políticas públicas que estimulen la inclusión de los desmovilizados al mercado laboral, de forma subordinada o independiente (Roldán Castellanos, 2010, p. 1).

Es justamente de esta premisa que puede soportarse la idea de que la contratación estatal fomente en el sector privado una idea de inclusión que se arraigue a través de incentivos desplegados de forma óptima en todos los procesos de selección de contratistas emprendidos por la Administración. En otras palabras, la contratación estatal cuenta con las herramientas, tanto jurídicas como materiales, para facilitar la introducción de preceptos que direccionen la reintegración de grupos con la intención de incorporarse a la sociedad, disminuir las barreras y hostilidades surgidas en el seno de la sociedad, y, como instrumento para atender los requerimientos legales y constitucionales que existen y se han consolidado bajo el postulado del Estado moderno. 
Según la Organización para la Cooperación y el Desarrollo Económico - OCDE, la contratación estatal representa cerca del $15 \%$ del producto interno bruto mundial y que, en Colombia, la Agencia Nacional de Contratación Pública, Colombia Compra Eficiente, afirma que este tipo de contratación, para el 2014, fue cercana al 16\% del PIB nacional, es decir, 115 billones de pesos (Rojas Rojas, y otros, 2016, p. 12).

Sin desvirtuar el objeto primario y fundamental de la contratación pública, entendido como el abastecimiento de obras, servicios y bienes; la realidad es que, por su influencia en el mercado, ésta puede ser utilizada como instrumento capaz de incidir el comportamiento del sector privado y garantizar objetivos que respondan a políticas públicas, leyes e incluso principios constitucionales.

La contratación estatal puede estimular el acceso de excombatientes a empleos formales y estables, entendiendo por éstos un derecho que por sus implicaciones sociales resulta esencial e innegociable y representa un desarrollo directo del concepto de dignidad humana y de la obligación estatal respecto a la sobrevivencia del ciclo económico del país.

La Administración no solo debe garantizar el acceso al trabajo en condiciones óptimas, sino que frente a las dificultades que se pueden evidenciar, debe hacer uso de todos los medios a su alcance para procurar un proceso de reintegración que no conlleve mayores traumatismos para los excombatientes, siguiendo los valores, fines, y objetivos normativos que desarrollan entre otras, el principio de igualdad que marca un hito importante en este proceso.

En la medida en que el trabajo constituye un factor de integración social, debe entenderse la inserción laboral de aquellos más desfavorecidos (e incluso discriminados, como lo son los desmovilizados) desde dos aspectos: como un fin y como un medio en sí mismo. Un fin pues su objetivo final es darle al trabajador desfavorecido la posibilidad de acceder a estructuras normales y legales de empleo, y un medio a través del cual este sea considerado, reintegrado y autoconsiderado como parte de la sociedad (Cháves Ávila \& Sajardo Moreno, 1999).

Lo contrario supondría, discriminación que bajo ningún respecto debería encontrar respaldo estatal, pues se estaría frente a conductas desplegadas de forma consciente o inconsciente por parte de los empleadores que limitan la escogencia de una persona apta, sin razones objetivas, para un trabajo particular. La inclusión de cláusulas sociales en los contratos públicos celebrados por la Administración, se revisten de importantes características que permiten resultados benéficos en el tránsito de los reinsertados a la vida social. Asimismo, es necesario establecer que, por sus repercusiones en las dimensiones humanas, el derecho al trabajo, adquiere gran relevancia tanto de forma autónoma como por conexidad con derechos fundamentales.

Es un deber de los servidores públicos instaurar en todas las esferas de la actividad (incluyendo por supuesto la contractual) un marco garante e integrador, capaz de desarrollar el núcleo esencial de este derecho. De ahí, que mediante Sentencia de Constitucionalidad C-251 de $1997^{7}$, el Magistrado Ponente Alejandro Martínez Caballero expresara:

La forma de Estado social de Derecho implica que las autoridades no solo deben garantizar a las personas esferas libres de interferencia ajena, sino también unas

\footnotetext{
${ }^{7}$ C-251 de 1997, Por medio de la cual se realizó el control automático de constitucionalidad de la Ley 319 de 1996, por medio de la cual se aprueba el protocolo acicional de la convención americana sobre derechos humanos, en materia de derechos económicos, sociales y culturales "protocolo de San Salvador", suscrito en San Salvador el 17 de noviembre de 1988.
} 
condiciones materiales mínimas que les posibiliten una existencia digna, las cuales identificó con el contenido mínimo de los $\mathrm{DESC}^{8}$, a los cuales pertenece al derecho al trabajo.

Ahora bien, el Estado no puede, ni debe interferir en la libertad de configuración de empresa y en las iniciativas privadas que quieran desplegarse de forma legal desde el sector privado. Así lo ha entendido la Corte Constitucional cuando se pronuncia estableciendo que el derecho al trabajo se circunscribe a la libertad de contratación con la que cuenta el empresario y que le permite desarrollar la facultad de elegir, de acuerdo a los parámetros que considere adecuados, al aspirante que mejor llene sus expectativas, aun cuando dicha elección obedezca a particulares circunstancias (Cfr. Cconst, C-408/2004, M.P. Alfredo Beltrán Sierra \& Cconst, t008/1992, M.P Simón Rodríguez Rodríguez \& Jaime Sanín Greiffenstein), como las propuestas en este documento.

Lo anterior resulta relevante en el entendido de que, si bien el Estado no podría limitar esta libertad, si puede proponer una serie de incentivos idóneos para fomentar la inclusión laboral de colectivos en condiciones de protección especial, lo que en el ámbito de la contratación estatal puede llegar a representar la especialización de los sectores, mayor competencia en el proceso de compra y mejores beneficios para la Administración.

Bajo esta perspectiva, la compra pública se configura como un medio idóneo para que el Estado, en su papel de agente económico que demanda bienes y servicios, intervenga y permita el acceso y la estabilidad laboral de personas pertenecientes a grupos guerrilleros con intenciones de reinsertarse en la sociedad; cuyos fundamentos se aferran a los postulados del bloque de constitucionalidad, a las leyes, reglamentos y políticas públicas cuyo enfoque otorga un papel protagónico al desarrollo de los Derechos Económicos, Sociales y Culturales como un desarrollo importante de las dimensiones interconectadas de la persona y de la sociedad misma.

El cierre de brechas es determinante como principio fundamental que debe guiar las labores del Estado con el propósito de corregir las desigualdades existentes, promover la inclusión y la participación, propiciar ambientes seguros para el ejercicio de las libertades fundamentales, incrementar el acceso a los servicios de justicia de las comunidades en contextos conflictivos, y garantizar a las personas o grupos en situación de desventaja el goce efectivo, gradual y progresivo, de sus derechos sociales fundamentales (Gobierno de Colombia, 2014, p. 11).

Debe mencionarse, frente al conflicto armado interno que los empresarios colombianos han producido respuestas variadas que difieren en cuanto al campo de acción, al impacto esperado, la escala y el diagnóstico que las subyace. Las respuestas difieren también en cuanto al grado de activismo que implican y si son fruto de decisiones individuales (como la fuga o la pasividad) o colectivas. En particular, aquellas dirigidas a la superación del conflicto por medio de proyectos productivos regionales o por medio del apoyo a los esfuerzos de negociación se distinguen de las demás por representar esfuerzos de cooperación y coordinación entre varios actores empresariales. Sólo la última respuesta apoyar las negociaciones de paz- incluye una significativa presencia gremial (Rettberg, 2002).

Se configura una nueva tarea para la Administración donde se dispongan determinadas cláusulas en los procesos contractuales desarrollando un esquema ejemplificador en la sociedad.

\footnotetext{
${ }^{8}$ La Sentencia T-025 de 2004 se pronunció definiéndo los derechos Económicos, Sociales y Culturales como "aquello necesario para proporcionar unas condiciones mínimas de vida digna, lo que exige del Estado social de derecho un esfuerzo mayor para su construcción, dentro de las posibilidades que estén a su alcance".
}

Revista de Direito Brasileira | São Paulo, SP | v. 17 | n. 7 | p. 63 - 75 | Mai./Ago. 2017 
La redacción de cláusulas que permitan solucionar objetivamente los casos de empate dentro de los procesos contractuales emprendidos por la Administración y que constituyan obligaciones de ejecución se presentan como iniciativas accesibles con la potencialidad de fomentar comportamientos benéficos en el sector privado.

Debe indicarse, sin embargo, que, aunque existe un desarrollo conceptual y legal formal de dicha etapa de reintegración, la realidad es que, como se mencionó previamente, de más de los 35.000 desmovilizados apenas un poco más de 1.000 han logrado conseguir un empleo. Los desmovilizados no son unos desempleados cualesquiera; por el contrario, tienen una serie de características que hacen aún más compleja la búsqueda de un empleo y un sostenimiento estable. Aunque cuentan con el apoyo económico del Estado a través de la ACR, tienen un bajo nivel educativo y poca aceptación civil, lo cual dificulta su integración social (Roldán Castellanos, 2010, p. 114).

Las cláusulas sociales, deben entenderse como un instrumento que aporta soluciones prácticas para la satisfacción de objetivos constitucional y legalmente establecidos, que para el caso en concreto, permiten influenciar a los diversos sectores empresariales para integrar laboralmente a los grupos de excombatientes y redes de apoyo y así fortalecer la fase de reintegración.

Milton Friedman dijo que la principal función de las empresas es hacer utilidades, y Michael Porter prueba que las empresas que más prosperan son las que invirtiendo sostenidamente en su entorno. El sector privado, sin sustituir al Estado, y en el contexto de los acuerdos de paz, puede contribuir a generar riqueza y equidad social. Y ese sería un gran legado. Para alcanzarlo, es necesario que los accionistas, directivos y empleados de las compañías asimilen dentro de su cultura y mentalidad que cualquier decisión de negocios tiene una valoración ética, una valoración cívica y un impacto en la comunidad (Pearl, 2015, p. 312).

El eje central de propuesta desarrollada lo constituye la inclusión de cláusulas sociales que a través de criterios de adjudicación, desempate o imposición de obligaciones de ejecución fomente el interés en los empresarios por dirigir su oferta laboral a desmovilizados interesados en reintegrarse a la sociedad.

Dentro de los elementos determinantes, debe tenerse especial atención en la configuración de los pliegos de condiciones, en los cuales debe exponerse de forma clara las características que quieran integrarse al contrato a celebrar, lo cual dotará de un carácter de legalidad todo el procedimiento.

\section{BIBLIOGRAFÍA}

\section{Doctrina}

DEPARTAMENTO NACIONAL DE PLANEACIÓN. 2014. Bases del Plan Nacional de Desarrollo 2014-2018, Bogotá: Gobierno de Colombia.

GIMENO, F. J. (2013). Compra pública estratégica. En S. C. Amoedo, C. C. Aymerich, G. G. Barrio, B. M. Bernal, A. A. Domínguez, F. J. Gimen. Rodr, Contratación pública estratégica, págs. 45-75. Pamplona: Aranzadi. 
MEJÍA GÓMEZ, L.F. 2014. La reintegración social y económica de los grupos armados ilegales en Colombia: reflexiones a partir de la trayectoria de nueve excombatientes. Bogotá: Universidad del Rosario.

NUSSIO, E. 2012. La vida después de la desmovilización. Percepciones, emociones y estrategias de ex paramilitares en Colombia. Bogotá: Departamento de Ciencia Política, Facultad de Ciencias Sociales, Universidad de los Andes.

PEARL, F. 2015. Hacia la modernidad. En S.C. Varios. Reconciliación. El gran desafío de Colombia. (págs. 311-314). Bogotá: Semana Libros.

REYES RODRÍGUEZ, C. 2015. Del individualismo a lo colectivo En S.C. Varios. Reconciliación. El gran desafío de Colombia. (págs. 230-232). Bogotá: Semana Libros.

ROLDÁN CATELLANOS, L. 2010. La inclusión laboral de los desmovilizados del conflicto en Colombia: auténtico mecanismo emancipador de la violencia en Colombia. Bogotá: Pontificia Universidad Javeriana.

ROJAS ROJAS, E., ZUBIETA CASAS, F., RODRÍGUEZ CASA, L., GARCÍA URREGO, L. O., CASTRO RIVEROS, M., \& FRANCO LEMUS, M. A. 2016. Modelo de Inteligencia de Mercado. Hacia entidades públicas ejemplares. Bogotá: Ministerio de Agricultura.

SPEAR, J. 2002. Disarmament and Demobilization. Ending Civil wars. London: Boulder London.

TORRES MENA, J. 2015. Contratación pública: Cláusulas Sociales. San Cristóbal de la Laguna: Universidad de la Laguna.

\section{Revistas}

CARAMÉS, A. (2006). Análisis de los programas de Desarme, Desmovilización y Reintegración existentes en el mundo durante 2005. Escola de Cultura de Pau y Agencia Española de Cooperación Internacional.

COLLIN (et al), G. (2004). Desarme, Desmovilización y Reintegración. Guía Teórica y Práctica. Suecia: Centro Pearson para el Mantenimiento de Paz, Cooperación Técnica Alemana y el Centro Noruego.

FISAS, V. (2011). Introducción al Desarme, Desmovilización y Reintegración de excombatientes. Escola de Cultura de Pau (epc), Quaderns de Construcció de Pau. N. 24.

Fundación ideas para la paz. (2014). Fin del conflicto: desarme, desmobilización y reintegraciónDDR. Boletín de paz No 33- Negociaciones de paz, 1-53.

HÖGBLADH, S. 2011. Peace Agreements 1975-2011-Updating the UCDP Peace Agreement Dataset. States in armed conflict, 39-56.

MEDINA JURADO, P. (mar-jun de 2010). Las cláusulas sociales en la contratación pública: retos y perspectivas. Revista Cemci (7). 
NUSSIO, E. (2009). ¿Reincidir o no? Conceptos de la literatura internacional aplicados al desarme, desmovilización y reintegración de las Autodefensas Unidas de Colombia. ¿La paz posible? Aproximaciones interdisciplinarias, Revista Pensamiento Jurídico (26).

RETTBERG, A. Administrando la adversidad: respuestas empresariales al conflicto colombiano. Revista Colombia Internacional. Obtenido en: https://colombiainternacional.uniandes.edu.co/view.php/411/1.php.

\section{Publicaciones electrónicas}

CHÁVES ÁVILA, R., \& SAJARDO MORENO, A. 2013. Obtenido de Universidad de Valencia: www.uv.es/sajardo/RVEA-insercion-laboral.pdf

\section{Jurisprudencia}

Colombia. Corte Constitucional. Sentencia C-251 de 1997, de 28 de mayo. Copia tomada directamente de esa corporación.

Sentencia C-408 de 2004, de 4 de mayo. Copia tomada directamente de esa corporación. . Sentencia T-025 de 2004, 22 de enero. Copia tomada directamente de esa corporación. 